\title{
Some comments on stochastic orders and posets
}

\author{
María Concepción López-Díaz ${ }^{1}$ and Miguel López-Díaz ${ }^{2}$
}

\begin{abstract}
In this paper we review some relations between partially ordered sets and stochastic orders. We focus our attention on analyzing if the property of being order-isomorphic is transferred from partially ordered sets to the stochastic orders generated by such partially ordered sets.
\end{abstract}

\section{Introduction}

The aim of this manuscript is to summarize some relations between partially ordered sets and stochastic orders defined by means of such ordered sets.

The theory of ordered sets plays an important role in many mathematical fields, as algebra, analysis, graph theory, combinatorics (see for instance [10], [1], [28]), [23]), etc. It is applied in different areas as computer science, coding theory, cryptography, (see for instance [13], [6], [22]), etc., as well as being by itself a remarkable area of interest for researchers.

Consider $(\mathcal{X}, \preceq \mathcal{X})$ a poset (partially ordered set), that is, $\mathcal{X}$ is a set and $\preceq \mathcal{X}$ is a binary relation defined on $\mathcal{X}$ which satisfies the reflexivity, transitivity and antisymmetric properties. Some of the most useful concepts related to ordered sets are the following.

A subset $U \subset \mathcal{X}$ is said to be an upper set if given $x_{1}, x_{2} \in \mathcal{X}$ with $x_{1} \in U$ and $x_{1} \preceq \mathcal{X} x_{2}$, then $x_{2} \in U$.

An upper quadrant set is a subset of $\mathcal{X}$ of the form $Q_{\bar{x}}^{\preceq \mathcal{X}}=\{z \in \mathcal{X} \mid x \preceq \mathcal{X}$ $z$ \}, with $x \in \mathcal{X}$. We will denote by $\mathcal{Q}^{\preceq \mathcal{X}}$ the class of upper quadrant sets determined by the partial order $\preceq \mathcal{X}$ on $\mathcal{X}$. Note that any upper quadrant set is an upper set.

Let $x_{1}, x_{2} \in \mathcal{X}$. We will say that $x_{2}$ covers $x_{1}$ if $x_{1} \preceq \mathcal{X} x_{2}, x_{1} \neq x_{2}$ and there is not $x_{3} \in \mathcal{X}, x_{1} \neq x_{3} \neq x_{2}$, with $x_{1} \preceq \mathcal{X} x_{3} \preceq \mathcal{X} x_{2}$.

\footnotetext{
${ }^{1}$ Departamento de Matemáticas, Universidad de Oviedo, Spain, cld@uniovi.es ${ }^{2}$ Departamento de Estadística e I.O. y D.M., Universidad de Oviedo, Spain, mld@uniovi.es
} 
In the case where $\mathcal{X}$ is a finite set, the Hasse diagram of the poset $(\mathcal{X}, \preceq \mathcal{X})$ is a directed graph with vertices set $\mathcal{X}$ and an edge from $x$ to $y$ if $y$ covers $x$.

To construct a Hasse diagram we will draw the points of $\mathcal{X}$ in the plane such that if $x_{1} \preceq \mathcal{X} x_{2}$, the point for $x_{2}$ has a larger $y$-(vertical) coordinate than the point for $x_{1}$.

Hasse diagrams are very useful representations of ordered sets which describe the order relations between elements in a graphical way.

Mappings from an ordered set to another ordered set which preserve the order relations, play a remarkable role in order theory. These mappings are said to be order-preserving mappings.

Let $\left(\mathcal{X}, \preceq_{\mathcal{X}}\right)$ and $(\mathcal{Y}, \preceq \mathcal{Y})$ be posets. A mapping $\phi: \mathcal{X} \rightarrow \mathcal{Y}$ is said to be order-preserving if for any $x_{1}, x_{2} \in \mathcal{X}$ with $x_{1} \preceq \mathcal{X} x_{2}$, we have that $\phi\left(x_{1}\right) \preceq \mathcal{Y} \phi\left(x_{2}\right)$. In particular, if $(\mathcal{X}, \preceq \mathcal{X})$ is a poset, a mapping $f: \mathcal{X} \rightarrow \mathbb{R}$ is said to be $\preceq \mathcal{X}$-preserving if for any $x_{1}, x_{2} \in \mathcal{X}$ with $x_{1} \preceq \mathcal{X} x_{2}$, we have that $f\left(x_{1}\right) \leq f\left(x_{2}\right)$. Note that the class of mappings which are $\preceq \mathcal{X}$-preserving is the class of order-preserving mappings when we consider the posets $(\mathcal{X}, \preceq \mathcal{X})$ and $(\mathbb{R}, \leq)$, $\leq$ being the usual order on the real line.

A very interesting class of order-preserving mappings is the class of bijective order-preserving maps. Let $(\mathcal{X}, \preceq \mathcal{X})$ and $(\mathcal{Y}, \preceq \mathcal{Y})$ be posets. A mapping $\phi: \mathcal{X} \rightarrow \mathcal{Y}$ is said to be an order-isomorphism if:

i) $\phi$ is order-preserving,

ii) there exists $\phi^{-1}: \mathcal{Y} \rightarrow \mathcal{X}$ inverse of $\phi$,

iii) $\phi^{-1}$ is order-preserving.

Clearly a mapping $\phi: \mathcal{X} \rightarrow \mathcal{Y}$ is an order-isomorphism if and only if

i) $\phi$ is bijective,

ii) for all $x_{1}, x_{2} \in \mathcal{X}$, it holds that $x_{1} \preceq \mathcal{X} x_{2}$ if and only if $\phi\left(x_{1}\right) \preceq \mathcal{Y} \phi\left(x_{2}\right)$.

Two posets $(\mathcal{X}, \preceq \mathcal{X})$ and $(\mathcal{Y}, \preceq \mathcal{Y})$ are said to be order-isomorphic if there exists an order-isomorphism $\phi: \mathcal{X} \rightarrow \mathcal{Y}$.

Note that two order-isomorphic sets are indistinguishable for the ordered sets theory, because they have the same order structure.

The reader is referred, for instance, to [14], [26] and [27] for an introduction to the theory of ordered sets.

In probabilistic and statistic frameworks, one of the main aims is to compare random magnitudes in accordance with an appropriate criterion. Ordered sets of probabilities have a great importance in this context. The study of such ordered sets involves the analysis of theoretical and applied problems. Pre-orders on sets of probabilities are called stochastic orders (see for instance [4], [25] and [29]). Stochastic orders have been successfully applied in areas like medicine, ecology, veterinary science, biology, economics, quality control theory, shape analysis, communications, etc (see for instance [2], [7], [8], [19], [20], [21], [16], [25], [17], [5], [30], [9], etc).

A stochastic order is said to be integral if there exists a class of real measurable mappings satisfying that $P \preceq Q$ when 


$$
\int_{\mathcal{X}} f d P \leq \int_{\mathcal{X}} f d Q
$$

for all $f$ in such a class, such that the above integrals exist, $P$ and $Q$ being probabilities. That class of mappings is said to be a generator of the order. We should note that there could be different generators of the same stochastic order (the reader is referred to [24] and Chapter 2 of [25] for integral stochastic orderings).

It is possible to define a stochastic order on the class of probabilities associated with a set endowed with a partial order by means of the class of preserving mappings as follows (see for instance [15] and [25]).

Let $(\mathcal{X}, \preceq \mathcal{X})$ be a poset. On $\mathcal{X}$ consider a $\sigma$-algebra $\mathcal{A}$. Let $\mathcal{F}^{\mathcal{X}}$ stand for the class of real measurable $\preceq \mathcal{X}$-preserving mappings. Let $\mathcal{P}_{\mathcal{X}}$ denote the set of probabilities associated with the measurable space $(\mathcal{X}, \mathcal{A})$. Define a pre-order $\left(\preceq_{\mathcal{X} g}\right)$ in that class by: let $P_{1}, P_{2} \in \mathcal{P}_{\mathcal{X}}$, then

$$
P_{1} \preceq \mathcal{X}_{g} P_{2} \quad \text { when } \quad \int_{\mathcal{X}} f d P_{1} \leq \int_{\mathcal{X}} f d P_{2}
$$

for all $f \in \mathcal{F}^{\mathcal{X}}$ for which both integrals exist.

Conditions for an integral stochastic order to be generated by a poset are studied in [18].

In some frameworks is quite useful to pay attention to the property of being order-isomorphic for stochastic orders generated by partially ordered sets, when such partially ordered sets are order-isomorphic.

\section{On order-isomorphisms of stochastic orders generated by posets}

In this section we consider the question proposed in Section 1 on the property of being order-isomorphic for posets and stochastic orders generated by such posets.

If two posets are order-isomorphic, then the stochastic orders generated by those posets are also order-isomorphic. It is interesting to remark that the converse is not true in general. We describe particular conditions under which the converse holds. These results are mainly included in [19].

Let us consider $(\mathcal{X}, \preceq \mathcal{X})$ a poset. We will denote by $\mathcal{B}_{\mathcal{X}}$ the $\sigma$-algebra generated by the class of upper quadrant sets, that is, $\mathcal{B}_{\mathcal{X}}=\sigma\left(\mathcal{Q}^{\preceq \mathcal{X}}\right)$.

The usual Borel $\sigma$-algebra on $\mathbb{R}$ will be denoted by $\mathcal{B}$.

The symbol $\mathcal{F}^{\mathcal{X}}$ will represent the set of mappings $f: \mathcal{X} \rightarrow \mathbb{R}$ which are measurable with respect to $\mathcal{B}_{\mathcal{X}}$ and $\mathcal{B}$, and $\preceq \mathcal{X}$-preserving.

On the other hand, $\mathcal{P}_{\mathcal{X}}$ will stand for the set of probability measures on the measurable space $\left(\mathcal{X}, \mathcal{B}_{\mathcal{X}}\right)$. Moreover, $\mathcal{P}_{\mathcal{X}}^{0}$ will denote the subset of $\mathcal{P}_{\mathcal{X}}$ composed by degenerated probabilities, that is, 
$\mathcal{P}_{\mathcal{X}}^{0}=\left\{P_{x} \in \mathcal{P}_{\mathcal{X}} \mid x \in \mathcal{X}, P_{x}(B)=1\right.$ if $x \in B, P_{x}(B)=0$ otherwise, $\left.B \in \mathcal{B}_{\mathcal{X}}\right\}$.

The class $\mathcal{F}^{\mathcal{X}}$ of all measurable $\preceq \mathcal{X}$-preserving mappings generates a stochastic order on $\mathcal{P}_{\mathcal{X}}$, denoted by $\preceq \mathcal{X}_{g}$, as follows: if $P_{1}, P_{2} \in \mathcal{P}_{\mathcal{X}}$, then

$$
P_{1} \preceq \mathcal{X}_{g} P_{2} \quad \text { when } \quad \int_{\mathcal{X}} f d P_{1} \leq \int_{\mathcal{X}} f d P_{2}
$$

for all $f \in \mathcal{F}^{\mathcal{X}}$ for which both integrals exist.

Note that $\left(\mathcal{P}_{\mathcal{X}}, \preceq_{\mathcal{X} g}\right)$ is a poset. Reflexivity and transitivity are obvious. Now if $P_{1} \preceq \mathcal{X}_{g} P_{2}$ and $P_{2} \preceq \mathcal{X}_{g} P_{1}$, since $I_{U} \in \mathcal{F}^{\mathcal{X}}, I_{U}$ being the indicator function of $U$, then $P_{1}(U)=P_{2}(U)$ for all $U \in \mathcal{A}^{\preceq}$, where $\mathcal{A}^{\checkmark}$ is the class of all finite intersections of upper quadrant sets. Since $\sigma\left(\mathcal{A}^{\preceq}\right)=\sigma\left(\mathcal{Q}^{\preceq}\right)$ and $\mathcal{A}^{\preceq}$ is a $\pi$-system, then $P_{1}=P_{2}$ (see for instance [3], p.42), that is, $\preceq \mathcal{X}_{g}$ satisfies the antisymmetric property.

The following result can be found in [12]. We should note that the finiteness of $\mathcal{X}$ is essential in that result.

Let $(\mathcal{X}, \preceq \mathcal{X})$ be a poset with $\mathcal{X}$ finite. Let $P_{1}, P_{2} \in \mathcal{P}_{\mathcal{X}}$, then $P_{1} \preceq \mathcal{X}_{g} P_{2}$ if and only if $P_{1}(U) \leq P_{2}(U)$ for any $U$ upper set.

If $\mathcal{X}$ is not finite, an upper set does not necessarily belong to $\mathcal{B}_{\mathcal{X}}$, and so the above result does not hold.

Now let us consider order-isomorphisms. Let $(\mathcal{X}, \preceq \mathcal{X})$ and $(\mathcal{Y}, \preceq \mathcal{Y})$ be posets and let $\phi: \mathcal{X} \rightarrow \mathcal{Y}$ be an order-isomorphism. It is not hard to prove that $\phi$ is measurable with respect to the $\sigma$-algebras $\mathcal{B}_{\mathcal{X}}$ and $\mathcal{B}_{\mathcal{Y}}$.

In relation to the property of being order-isomorphic, the following result is obtained in [19].

Let $\left(\mathcal{X}, \preceq_{\mathcal{X}}\right)$ and $(\mathcal{Y}, \preceq \mathcal{Y})$ be order-isomorphic posets. Then the posets $\left(\mathcal{P}_{\mathcal{X}}, \preceq_{\mathcal{X} g}\right)$ and $\left(\mathcal{P}_{\mathcal{Y}}, \preceq_{\mathcal{Y}_{g}}\right)$ are order-isomorphic.

We should remark that an order-isomorphism $\nabla$ between $\left(\mathcal{P}_{\mathcal{X}}, \preceq_{\mathcal{X} g}\right)$ and $\left(\mathcal{P} \mathcal{Y}, \preceq \mathcal{Y}_{g}\right)$ could be defined by means of an order-isomorphism $\phi$ between $(\mathcal{X}, \preceq \mathcal{X})$ and $(\mathcal{Y}, \preceq \mathcal{Y})$. This fact is quite useful in many applications.

Example 1. (from [19]) Consider the posets $(\mathcal{X}, \preceq \mathcal{X})$ and $(\mathcal{Y}, \preceq \mathcal{Y})$ whose Hasse diagrams (taken from [27]) are given in Figure 1.
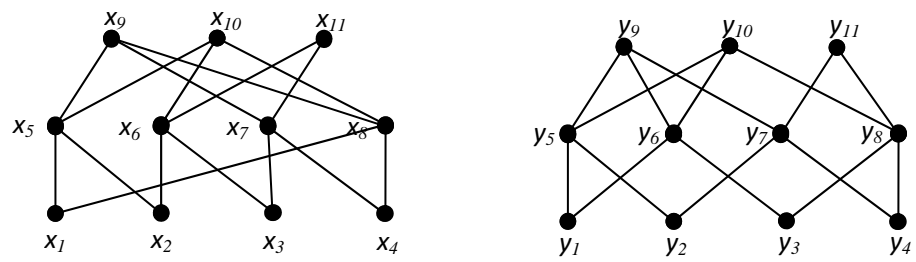

Fig. 1 Hasse diagrams in Example 1 
Let $\phi: \mathcal{X} \rightarrow \mathcal{Y}$ be the mapping such that $\phi\left(x_{1}\right)=y_{1}, \phi\left(x_{2}\right)=y_{2}$, $\phi\left(x_{3}\right)=y_{4}, \phi\left(x_{4}\right)=y_{3}, \phi\left(x_{5}\right)=y_{5}, \phi\left(x_{6}\right)=y_{7}, \phi\left(x_{7}\right)=y_{8}, \phi\left(x_{8}\right)=y_{6}$, $\phi\left(x_{9}\right)=y_{10}, \phi\left(x_{10}\right)=y_{9}$ and $\phi\left(x_{11}\right)=y_{11}$. Then $\phi$ is an order-isomorphism and the posets $(\mathcal{X}, \preceq \mathcal{X})$ and $(\mathcal{Y}, \preceq \mathcal{Y})$ are order-isomorphic. As a consequence, we conclude that the posets $\left(\mathcal{P}_{\mathcal{X}}, \preceq \mathcal{X}_{g}\right)$ and $\left(\mathcal{P}_{\mathcal{Y}}, \preceq \mathcal{Y}_{g}\right)$ are order-isomorphic.

The converse of the result above is not true in general, as the following example shows (see [19]).

Example 2. Consider the sets $\mathcal{X}=\left\{x_{1}, x_{2}, x_{3}\right\}$ and $\mathcal{Y}=\left\{y_{1}, y_{2}\right\}$, and the posets $(\mathcal{X}, \preceq \mathcal{X})$ and $(\mathcal{Y}, \preceq \mathcal{Y})$ with the Hasse diagrams given in Figure 2 .

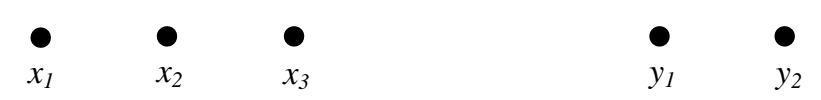

Fig. 2 Hasse diagrams in Example 2

That posets are not order-isomorphic since $|\mathcal{X}| \neq|\mathcal{Y}|$.

Note that $\mathcal{X}$ and $\mathcal{Y}$ are finite. Then, by means of the result in [12] given above, if $P_{1}, P_{2} \in \mathcal{P}_{\mathcal{X}}$ with $P_{1} \neq P_{2}$, we have that neither $P_{1} \preceq \mathcal{X}_{g} P_{2}$ nor $P_{2} \preceq \mathcal{X}_{g} P_{1}$ are satisfied, and the same happens with the probabilities of $\mathcal{P}_{\mathcal{Y}}$. As a consequence, if we define a bijection between $\mathcal{P}_{\mathcal{X}}$ and $\mathcal{P}_{\mathcal{Y}}$, it will be an order-isomorphism. It is not hard to prove that there exists a bijection between both sets (see for instance [11]), and so $\left(\mathcal{P}_{\mathcal{X}}, \preceq \mathcal{X}_{g}\right)$ and $\left(\mathcal{P}_{\mathcal{Y}}, \preceq \mathcal{Y}_{g}\right)$ are order-isomorphic.

Now the question is to find conditions under which an order-isomorphism between $\left(\mathcal{P}_{\mathcal{X}}, \preceq \mathcal{X}_{g}\right)$ and $\left(\mathcal{P}_{\mathcal{Y}}, \preceq \mathcal{Y}_{g}\right)$ implies the existence of an orderisomorphism between $(\mathcal{X}, \preceq \mathcal{X})$ and $(\mathcal{Y}, \preceq \mathcal{Y})$. Some of such conditions were proved in [19].

The following result holds.

Let $(\mathcal{X}, \preceq \mathcal{X})$ and $(\mathcal{Y}, \preceq \mathcal{Y})$ be posets, consider the posets $\left(\mathcal{P}_{\mathcal{X}}, \preceq \mathcal{X}_{g}\right)$ and $\left(\mathcal{P}_{\mathcal{Y}}, \preceq \mathcal{Y}_{g}\right)$. Let $\nabla: \mathcal{P}_{\mathcal{X}} \rightarrow \mathcal{P}_{\mathcal{Y}}$ be an order-isomorphism such that $\nabla\left(\mathcal{P}_{\mathcal{X}}^{0}\right)=$ $\mathcal{P}_{\mathcal{Y}}^{0}$, then $(\mathcal{X}, \preceq \mathcal{X})$ and $(\mathcal{Y}, \preceq \mathcal{Y})$ are order-isomorphic.

Another condition is provided by the following concept in [19], which in conjunction with an order-isomorphism between $\left(\mathcal{P}_{\mathcal{X}}, \preceq \mathcal{X}_{g}\right)$ and $\left(\mathcal{P}_{\mathcal{Y}}, \preceq \mathcal{Y}_{g}\right)$, lead to an order-isomorphism between $(\mathcal{X}, \preceq \mathcal{X})$ and $(\mathcal{Y}, \preceq \mathcal{Y})$. Let $(\mathcal{X}, \preceq \mathcal{X})$ be a poset and $I \subset \mathcal{X}$. A mapping $\Upsilon: I \rightarrow \mathcal{X}$ is said to conserve $\preceq \mathcal{X}$ in each point separately, when given $x_{1}, x_{2} \in I$, if one of the following relations holds: $x_{1} \preceq \mathcal{X} x_{2}, \Upsilon\left(x_{1}\right) \preceq \mathcal{X} x_{2}, x_{1} \preceq \mathcal{X} \Upsilon\left(x_{2}\right)$, then the three relations are satisfied simultaneously.

Given $(\mathcal{X}, \preceq \mathcal{X})$ a poset and $S$ a subset of $\mathcal{X}$, we will denote by $\bar{S}$ the complement of $S$ in $\mathcal{X}$, that is, $\bar{S}=\mathcal{X} \backslash S$.

The following result gives a new condition involving mappings which conserve orders in each point separately (see [19]). 
Let $(\mathcal{X}, \preceq \mathcal{X})$ and $(\mathcal{Y}, \preceq \mathcal{Y})$ be posets, let us consider the posets $\left(\mathcal{P}_{\mathcal{X}}, \preceq \mathcal{X}_{g}\right)$ and $\left(\mathcal{P} \mathcal{Y}, \preceq \mathcal{Y}_{g}\right)$. Let $\nabla: \mathcal{P}_{\mathcal{X}} \rightarrow \mathcal{P} \mathcal{Y}$ be an order-isomorphism. Let us define the

sets $L=\nabla^{-1}\left(\mathcal{P}_{\mathcal{Y}}^{0}\right) \cap \overline{\mathcal{P}_{\mathcal{X}}^{0}}$ and $M=\nabla\left(\mathcal{P}_{\mathcal{X}}^{0}\right) \cap \overline{\mathcal{P}_{\mathcal{Y}}^{0}}$. If $L$ and $M$ are nonempty sets and if there exists an order-isomorphism $\Omega: L \rightarrow M$ such that $\Omega^{-1} \circ \nabla$ : $\nabla^{-1}(M) \rightarrow \mathcal{P}_{\mathcal{X}}$ and $\nabla^{-1} \circ \Omega: L \rightarrow \mathcal{P}_{\mathcal{X}}$ conserve $\preceq_{\mathcal{X}}$ in each point separately, then the posets $(\mathcal{X}, \preceq \mathcal{X})$ and $(\mathcal{Y}, \preceq \mathcal{Y})$ are order-isomorphic.

The proof can be found in [19], together with an interesting application to the analysis of chemical components of seaweeds.

Acknowledgements The authors are indebted to the Spanish Ministry of Science and Innovation and Principado de Asturias since this research is financed by Grants MTM201122993, MTM2013-45588-C3-1-P, FC-15-GRUPIN14-101 and FC-15-GRUPIN14-142.

\section{References}

1. Arhippainen J, Kauppi J, Mattas J (2017) Order structure, multipliers, and Gelfand representation of vector-valued function algebras. Banach J Math Anal 11:207-222

2. Ayala G, López-Díaz MC, López-Díaz M, Martínez-Costa L (2012) Studying hypertension in ocular fundus images using Hausdorff dispersion ordering. Math Med Biol $2: 131-143$

3. Billingsley, P (1995) Probability and Measure. John Wiley \& Sons, New York

4. Belzunce F, Martínez-Riquelme C, Mulero J (2016) An Introduction to Stochastic Orders. Elsevier/Academic Press, Amsterdam

5. Blaszczyszyn B, Yogeshwaran D (2009) Directionally convex ordering of random measures, short noise fields, and some applications to wireless communications Adv Appl Prob 41:623-646

6. Brualdi RA, Graves JS, Lawrence KM (1995) Codes with a poset metric. Discrete Math 147:57-72

7. Carleos C, López-Díaz M (2010) An indexed dispersion criterion for testing the sexbiased dispersal of lek mating behavior of capercaillies. Environ Ecol Stat 17:283-301.

8. Carleos C, López-Díaz MC, López-Díaz M (2010) A stochastic order of shape variability with an application to cell nuclei involved in mastitis. J Math Imaging Vis 38:95-107

9. Carleos C, López-Díaz, MC, López-Díaz, M (2014) Ranking star-shaped valued mappings with respect to shape variability. J Math Imaging Vis 48:1-12

10. Cirulis J (2017) The diamond partial order for strong Rickart rings. Linear Multilinear Algebra 65:192-203

11. Dugundji J (1966) Topology. Allyn and Bacon, Boston

12. Giovagnoli A, Wynn HP (2008) Stochastic orderings for discrete random variables. Statist Prob Lett 78:2827-2835

13. Garg VK (2015) Introduction to lattice theory with computer science applications. John Wiley \& Sons, Hoboken

14. Harzheim E (2005) Ordered Sets. Advances in Mathematics 7. Springer, New York

15. Kamae T, Krengel U, O'Brien GL (1977) Stochastic partial orgering. Ann Probab 6:1044-1049

16. Kijima M, Ohnishi M (1999) Stochastic orders and their applications in financial optimization. Math Meth Oper Res 50:351-372 
17. López-Díaz M (2011) A test for the bidirectional stochastic order with an application to quality control theory. Appl Math Comput 217:7762-7771

18. López-Díaz MC, López-Díaz M (2012) When is an integral stochastic order generated by a poset? J Ineq Appl 2012:Art.265

19. López-Díaz MC, López-Díaz M (2013) On order-isomorphisms of stochastic orders generated by partially ordered sets with applications to the analysis of chemical components of seaweeds. MATCH Commun Math Comput Chem 69:463-486

20. López-Díaz MC, López-Díaz M (2013) A note on the family of extremality stochastic orders. Insur Math Econom 53:230-236

21. López-Díaz MC, López-Díaz M, Martínez-Fernández S (2017) A stochastic comparison of customer classifiers with an application to customer attrition in commercial banking Scand Actuar J 2017(7):606-627

22. Lu X, Wen Q, Wang L, Du J (2016) A lattice-based signcryption scheme without trapdoors. J Elect Inform Techy 38:2287-2293

23. McConville T (2017) Lattice structure of GridTamari orders. J Comb Theory A 148:27-56

24. Müller A (1997) Stochastic orders generated by integrals: a unified study. Adv Appl Probab 29:414-428

25. Müller A, Stoyan D (2002) Comparison Methods for Stochastic Models and Risks. John Wiley \& Sons, Chichester

26. Neggers J, Kim HS (1998) Basic Posets. World Scientific Pub, Singapore

27. Schröder BSW (2003) Ordered Sets, An Introduction. Birkhäuser, Boston

28. Sebastian Vadakkenveettil B, Unnikrishnan A, Balakrishnan K, Padinjare Pisharath Balakrishna R (2017) Morphological filtering on hypergraphs. Disc Appl Math 216:307-320

29. Shaked M, Shanthikumar JG (2007) Stochastic Orders. Springer, New York

30. Tepedelenlioglu C, Rajan A, Zhang Y (2011) Applications of stochastic ordering to wireless communications. IEEE Trans Wirel Comm 10: 4249-4257 